\title{
Type 2 Diabetes, Metabolic Syndrome and Lipids
}

\author{
Orit Pinhas-Hamiel \\ Pediatric Endocrinology and Diabetes Unit, Edmond and Lily Safra Children's Hospital, Sheba Medical Center, \\ Tel-Hashomer, Ramat-Gan and Maccabi Juvenile Diabetes Center, Raanana, Sackler School of Medicine, Tel- \\ Aviv University, Israel
}

\begin{abstract}
The complexity of the development of type 2 diabetes mellitus (T2DM) is exhibited in the story of the Oji-Cree people. Long-term surveillance of the offspring of mothers who were diagnosed with T2DM in their own childhood demonstrates the interaction between the changing environment, genetics, and antenatal factors.

This year we learnt of yet another group at risk for early-onset T2DM: children treated with growth hormone (GH). The results of the first long-term clinical trial to maintain glycemic control among adolescents with T2DM are worrisome. Even more worrisome are the findings of a computer model calculating the risk of morbidity and mortality for youngsters with T2DM. We learnt that the development of the metabolic syndrome (MetS) during adolescence can be predicted by natal and parental profiles. This is important since children with MetS are at risk for developing kidney stones. The hours of sleep and the quality of sleep contribute to the development of MetS.

New guidelines for universal screening for dyslipidemia in children were published this year. Studies in adults show that intensive statin treatment is associated with an increased risk of new-onset T2DM. It is comforting to know that there are new drugs to reduce LDL levels in the pipeline.
\end{abstract}

\section{Type 2 diabetes New paradigms}

\section{Obesity and type 2 diabetes mellitus in a birth cohort of First Nation children born to mothers with pediatric-onset type 2 diabetes}

Mendelson M, Cloutier J, Spence L, Sellers E, Taback S, Dean H

Section of Endocrinology and Metabolism, Department of Pediatrics, University of Manitoba, Winnipeg, Man.,

Canada

Pediatr Diabetes 2011;12:219-228

Background: Children who are born to mothers with pediatric-onset type 2 diabetes mellitus (T2DM) are exposed to a hyperglycemic intrauterine environment throughout pregnancy. The growth patterns and risk of T2DM in these offspring may be influenced by unique gene-environment interactions during intrauterine and postnatal life.

Methods: A cohort of offspring of First Nation mothers with onset of T2DM before age 18 years in Manitoba, Canada, was established. Height or length and weight at study entry and annually thereafter with fasting blood glucose in offspring aged $\geq 7$ years were measured. Birth and breastfeeding history were collected, as well as specific hepatic nuclear factor- $1 \alpha$ (HNF-1 $\alpha)$ G319S genotype of offspring at age 7 years.

Results: From July 2003 to April 2008, 76 offspring of 37 mothers were enrolled. 64\% (23/36) of the offspring aged 2-19 years were obese at initial assessment. The rates of obesity remained constant throughout the 5 years. As of April 2008, 7/28 (25\%) of the offspring aged 7-19 years have diabetes including 6/14 (43\%) aged 10-19 years. Most offspring with diabetes (5/7, 71\%) were obese at diagnosis. All of the 7 offspring with diabetes have one or two copies of the G319S polymorphism.

Conclusions: The prevalence of T2DM in this cohort of offspring of First Nation women with pediatricT2DM is the highest ever reported in children. Obesity is an important postnatal risk factor for T2DM in this population and may result from a unique gene-environment interaction. 
This report, which tells us the story of the Oji-Cree people, is a result of 'The Next Generation Project' that began in 2003. The project has established long-term surveillance of the offspring of Aboriginal mothers who were diagnosed with T2DM during their own childhood or adolescence. The prevalence of T2DM in the Canadian Oji-Cree population ranks among the highest in the world. They were the first population to experience T2DM in a pediatric age group. Subjects from the Oji-Cree population were found to have a private polymorphism of the hepatic nuclear factor-1 $\alpha$ (HNF-1 $\alpha$ ) [1]. HNF-1 $\alpha$ is a transcriptional activator of many genes including insulin, albumin, $\alpha_{1}$-antitrypsin, and fibrinogen; it is expressed predominantly in the liver and the kidney, but also in the pancreas. Rare deleterious mutations in HNF-1 $\alpha$ occur in maturity-onset diabetes of the young type 3 (MODY-3). The Oji-Cree polymorphism in the HNF-1 $\alpha$ gene leads to an amino acid substitution of glycine to serine at codon 319 (G319 to S319). Carriers of a single copy of the S319 allele have about 3-fold higher risk of T2DM, whereas \$319/S319 homozygotes have a 120-fold higher risk. Moreover, the age of onset of T2DM is earlier with an increased number of the $\mathbf{S} 319$ alleles. This polymorphism occurs in about $8 \%$ of the general Oji-Cree population but it is present in about $40 \%$ of those who developed T2DM. Two generations ago the diagnosis of diabetes was virtually unknown among the Oji-Cree people, though the S319 mutation must have been present, thus HNF-1 $\alpha$ appears to be a susceptibility gene in subjects exposed to an abundance of food and limited physical activity.

In the current study, because of the high rates and the early onset of T2DM among the offspring, the authors suggest that the intrauterine exposure to maternal obesity and diabetes may have accelerated the progression of T2DM. Alteration of $\beta$-cell mass through prenatal programming of the fetus by epigenetic influences might possibly explain the high rate of early-onset diabetes in this cohort [2].

\section{Type 2 diabetes \\ New concerns}

\section{Prevalence and incidence of diabetes mellitus in GH-treated children and adolescents: analysis from the GeNeSIS observational research program}

Child CJ, Zimmermann AG, Scott RS, Cutler GB Jr, Battelino T, Blum WF

Lilly Research Laboratories, Erl Wood Manor, Windlesham, UK

cjc@lilly.com

J Clin Endocrinol Metab 2011;96:E1025-1034

Background: GH has an insulin antagonist effect, and GH treatment has therefore been suggested to impair glucose metabolism and increase risk of diabetes mellitus.

Methods: Data from 11,686 GH-treated patients in the Genetics and Neuroendocrinology of Short Stature International Study (GeNeSIS), a multinational observational study of children with growth disorders, were analyzed for diabetes incidence. Baseline diabetes prevalence was determined from a GH-naive subgroup. Prevalence and incidence (by standardized incidence ratio) were compared with results from patients aged less than 20 years in the US SEARCH for Diabetes in Youth study.

Results: Baseline type 1 diabetes (T1DM) prevalence per 1,000 persons was 4.92 (95\% CI 1.91-12.58) in GeNeSIS and 1.03 (0.97-1.10) in SEARCH for 0- to 9 year-olds, and 7.33 (4.20-12.77) and 2.99 (2.782.98), respectively, for 10- to 19-year-olds. There were no GeNeSIS cases of T2DM before GH initiation. During a median 1.8 years of GH treatment, diabetes standardized incidence ratios for US patients were $1.4(0.5-3.1)$ for T1DM and 8.5 (2.8-19.5) for T2DM, and for all patients was $1.4(0.7-2.4)$ for T1DM and 6.5 (3.3-11.7) for T2DM. Among the 11 patients with incident T2DM, preexisting risk factors for diabetes were identified in 10. Glucose concentrations normalized in 7 of 9 patients for whom glycemic status could be determined, 3 of whom continued GH therapy and 4 who discontinued.

Conclusion: The incidence of T2DM was higher in GH-treated children than the general population. Monitoring of glucose, before and periodically during GH treatment, is recommended for those with preexisting T2DM risk factors.

Besides regulating growth, $\mathrm{GH}$ has a major function in maintaining carbohydrate and lipid homeostasis which may have important relevance in conditions in which GH activity is high. When GH is 
secreted in excess, it acts directly to block insulin signaling, which then causes an elevation of glucose and insulin concentrations. Indeed impaired glucose intolerance, relative hyperinsulinemia, and overt T2DM are common features in acromegaly. Similarly, an increased incidence for T2DM in GH-treated children and adolescents was reported a decade ago [3]. In light of the rising prevalence of obesity in the general pediatric population and the trend toward higher $\mathrm{GH}$ doses, the objective of the study was to reevaluate the impact of GH treatment.

The Genetics and Neuroendocrinology of Short Stature International Study (GeNeSIS) is an openlabel multinational observational safety study. The findings show that children who received growth hormone treatment had more than 8-fold increased risk of developing T2DM compared with unselected children. Children with organic growth hormone deficiency had a higher incidence of T2DM than children with idiopathic growth hormone deficiency. The majority of patients who developed T2DM had preexisting risk factors for diabetes such as Turner syndrome, Prader-Willi syndrome, and obesity, as well as small for gestational age and growth hormone deficiency due to leukemia and irradiation. Among the 11 patients who developed T2DM, hyperglycemia resolved in 7 patients. It is noteworthy that an additional 13 children had abnormal glucose tolerance.

This is an observational study and the value of the analysis depends on the accurate reporting of all events by the participating physicians, thus the rates might be biased. However, it seems that clinicians need to notify parents about the risk of T2DM, especially those with high-risk factors. Attention to glucose metabolism, both before and during GH treatment, is warranted.

\section{Type 2 diabetes \\ Important for clinical practice}

\section{A clinical trial to maintain glycemic control in youth with type 2 diabetes}

TODAY Study Group, Zeitler P, Hirst K, Pyle L, Linder B, Copeland K, Arslanian S, Cuttler L, Nathan DM, Tollefsen S, Wilfley D, Kaufman F

N Engl J Med 2012;366:2247-2256

Background: Despite the increasing prevalence of T2DM in youth, there are few data to guide treatment. The efficacies of three treatment regimens to achieve durable glycemic control in children and adolescents with recent-onset T2DM were compared.

Methods: All eligible patients 10-17 years of age were treated with metformin (1,000 mg twice daily) during the run-in phase to attain a glycated hemoglobin level of less than $8 \%$, and were then randomly assigned to continued metformin alone, or metformin plus rosiglitazone ( $4 \mathrm{mg}$ twice daily) or metformin plus a lifestyle-intervention program focusing on weight loss through eating and activity behaviors. The primary outcome was loss of glycemic control, defined as a glycated hemoglobin level of at least $8 \%$ for 6 months or sustained metabolic decompensation requiring insulin.

Results: Of the 699 randomized participants (mean duration of T2DM, 7.8 months), 319 (45.6\%) reached the primary outcome over an average follow-up of 3.86 years. Rates of treatment failure were $51.7 \%$ (120/232), 38.6\% (90/233), and 46.6\% (109/234) for metformin alone, metformin plus rosiglitazone, and metformin plus lifestyle intervention, respectively. Metformin plus rosiglitazone was superior to metformin alone $(\mathrm{p}=0.006)$; metformin plus lifestyle intervention was intermediate but not significantly different to the other groups. Prespecified subgroup analyses showed differences in sustained effectiveness, with metformin alone least effective in non-Hispanic black participants and metformin plus rosiglitazone most effective in girls. Serious adverse events were reported in $19.2 \%$.

Conclusions: Monotherapy with metformin was associated with durable glycemic control in approximately half of children and adolescents with T2DM. The addition of rosiglitazone, but not an intensive lifestyle intervention, was superior to metformin alone.

The findings of this report are highly disappointing. Regardless of the therapy, about half of adolescents with T2DM failed to achieve adequate metabolic control of $\mathrm{HbA}_{1 \mathrm{c}}<8 \%$. The American Diabetes Association recommends target $\mathrm{HbA}_{1 \mathrm{c}}$ values $<7.5 \%$ for adolescents with T1DM aged 13-18 years. There is no reason to assume that a different target should be set for those with T2DM; on the con- 
trary, youth with T2DM have additional risk factors that put them at increased risk for morbidity. T2DM is becoming increasingly common among youth in parallel with rates of childhood obesity. Despite this trend, there have been only a limited number of clinical trials investigating pharmacotherapy for T2DM in youth. Therapeutic modalities remain extremely limited and there are insufficient data on their effectiveness in children and adolescents. Most of the recommendations for treating T2DM in childhood are extrapolated from adults [4]. This is why this study is so important. Although the addition of rosiglitazone improved primary outcomes, this group showed increased weight gain. In contrast, metformin plus lifestyle intervention decreased the percentage of overweight adolescents. Two more noteworthy findings were: serious adverse effects were reported in $19.2 \%$ of adolescents, and there was no difference in rate of loss of glycemic control between participants who adhered to their medication regimen and those who did not. If metformin monotherapy at maximal tolerated dose is insufficient, additional treatment must be sought. In adults, intensive treatment with insulin in newly diagnosed T2DM can result in sustained remission. Studies on the efficacy and safety of long-acting glucagon-like peptide-1 (GLP-1) analog in combination with metformin and a selective dipeptidyl peptidase IV (DPP-4) inhibitor are currently ongoing. Long-term clinical studies with insulin treatment for youth with T2DM are needed.

\section{Type 2 diabetes Food for thought}

\section{Estimated morbidity and mortality in adolescents and young adults diagnosed with type 2 diabetes mellitus}

Rhodes ET, Prosser LA, Hoerger TJ, Lieu T, Ludwig DS, Laffel LM

Division of Endocrinology, Children's Hospital Boston, Boston, MA, USA

erinn.rhodes@childrens.harvard.edu

Diabet Med 2012;29:453-463

Background: To estimate remaining life expectancy (RLE), quality-adjusted life years (QALY), causes of death and lifetime cumulative incidence of microvascular/macrovascular complications of diabetes for youths diagnosed with T2DM.

Methods: A Markov-like computer model simulated the life course for a hypothetical cohort of adolescents/young adults in the USA, aged 15-24 years, newly diagnosed with T2DM following either conventional or intensive treatment based on the UK Prospective Diabetes Study. Outcomes included RLE, QALYs, cumulative incidence of microvascular/macrovascular complications and causes of death.

Results: Compared with a mean RLE of 58.6 years for a 20 -year-old in the USA without diabetes, those with T2DM on conventional treatment had an average RLE of 43.09 years and 22.44 discounted QALYs. Intensive treatment increased RLE by 0.98 years and QALYs by 0.44 years, and lowered lifetime cumulative incidence of microvascular complications and mortality from microvascular complications (e.g. end-stage renal disease (ESRD) death 19.4 vs. $25.2 \%$ ). Approximately $5 \%$ with both treatments had ESRD within 25 years. Lifetime cumulative incidence of coronary heart disease (CHD) increased with longer RLE and greater severity of CHD risk factors. When incorporating disutility (loss in health-related quality of life), intensive treatment resulted in net loss of QALYs.

Conclusions: Adolescents/young adults with T2DM lose approximately 15 years from RLE and will experience severe, chronic complications of T2DM by their 40s. The net benefit of intensive treatment may be sensitive to preferences for treatment. A comprehensive management plan that includes early and aggressive control of cardiovascular risk factors is likely needed to reduce lifetime risk of CHD.

The onset of T2DM in adolescence appears to place the individual at very high risk for later major morbidity. The rate of progression of complications in adolescents with T2DM may be more rapid than in adolescents with T1DM. Understanding the true risks of early-onset T2DM on life expectancy may take decades. These authors used an alternative computer model to simulate a hypothetical course in adolescents with T2DM.

The authors estimated that an adolescent with T2DM on conventional treatment would be expected 
to survive until their early 60 s, compared the late 70 s in a healthy 20 -year-old in the USA. Moreover, QALYs were reduced to only 22 years, suggesting that they may experience severe complications of T2DM by their 40s. The concept of quality-adjusted life-years (QALYs) is key to understanding this report. QALY is a measure for survival that accounts for the suboptimal quality of life due to poor health. One year of life lived in perfect health equals 1 QALY, while 1 year of life lived in poor health equals less than 1, ranging in number down to zero for death. The computer model relied on several assumptions about rates of complications and the impact of intervention. Risks were calculated from existing studies in adolescents with T2DM, and the effects of intervention (intensive vs. conventional) were based on the United Kingdom Prospective Diabetes Study (UKPDS) of adults with T2DM. One can only hope that the reality is more encouraging than these estimates.

\title{
Metabolic syndrome New concerns
}

\section{Metabolic syndrome in obese adolescents is associated with risk for nephrolithiasis}

\author{
Tiwari R, Campfield T, Wittcopp C, Braden G, Visintainer P, Reiter EO, Allen HF \\ Department of Pediatrics, Baystate Medical Center/Tufts University School of Medicine, Boston, MA, USA \\ J Pediatr 2012;160:615-620 e2
}

Background: To examine the relationship between urinary $\mathrm{pH}$ and metabolic syndrome risk factors along with insulin resistance in obese adolescents, and to evaluate the relationship between other urinary stone-forming and -inhibiting markers and MetS.

Methods: 46 obese adolescents (mean age $14.6 \pm 2.0$ years; mean BMI $36 \pm 6.3 \mathrm{~kg} / \mathrm{m}^{2}$ ) were enrolled. 24 Hour and random urine samples were analyzed for urinary $\mathrm{pH}$, promoters of stone formation (uric acid, oxalate, and relative saturation ratio of calcium oxalate (RSR-CaOx)), and inhibitors of stone formation (citrate and osteopontin). Height, weight, blood pressure, and fasting lipid, insulin, and glucose levels were also collected.

Results: Random urine $\mathrm{pH}$ was inversely related to the number of MetS risk factors $(\mathrm{r}=-0.34 ; \mathrm{p}=0.02)$. RSR-CaOx was positively related to both the insulin resistance score $(r=0.38 ; p<0.01)$ and number of MetS risk factors $(\mathrm{r}=0.47 ; \mathrm{p}=0.001)$.

Conclusion: Decreased urinary $\mathrm{pH}$ and increased $\mathrm{RSR}-\mathrm{CaOx}$ were associated with MetS risk factors in obese adolescents.

Among adults the prevalence of nephrolithiasis is increasing in parallel with the rising prevalence of obesity, and the risk of nephrolithiasis is increased in adults with obesity, MetS, and T2DM [5]. Insulin resistance plays a key role in the development of renal stones, being associated with low urinary $\mathrm{pH}$, a potential cause of uric acid nephrolithiasis.

An increase in the number of children with nephrolithiasis has been documented in the last decade as well. In the current study, urine $\mathrm{pH}$ was unrelated to BMI Z-score, but pH declined with an increasing number of MetS traits in obese adolescents. The mean fresh urine $\mathrm{pH}$ was 5.23 in obese adolescents with 3 or more metabolic risk factors, compared with 6.12 in those with no risk factors. In addition, the relative saturation of calcium oxalate, a measure of the likelihood of precipitation of minerals in renal stones, was elevated in obese adolescents with more metabolic syndrome risk factors. These findings again show that the pathophysiological process of adult disease begins in childhood.

Michelangelo (1475-1564) suffered from recurrent urinary stones throughout his life and possibly died of fluid overload due to obstructive nephropathy. This may account for his interest in kidney function, evident in his poetry and drawings [6]. Most impressive is the mantle of the Creator in his painting of the Separation of Land and Water on the ceiling of the Sistine Chapel, which is in the shape of a bisected right kidney. His use of the renal outline in a scene representing the separation of solids (Land) from liquid (Water) suggests that Michelangelo was likely familiar with the anatomy and function of the kidney. 


\title{
Metabolic syndrome in adolescence: can it be predicted from natal and parental profile? The Prediction of Metabolic Syndrome in Adolescence (PREMA) study
}

\author{
Efstathiou SP, Skeva II, Zorbala E, Georgiou E, Mountokalakis TD \\ Center for Cardiovascular Disease Prevention, Hygeias Melathron Infirmary, Athens, Greece \\ stamatise@gmail.com \\ Circulation 2012:125:902-910
}

Background: There are well-established predisposing factors for the metabolic syndrome (MetS) in childhood or adolescence, but no specific risk profile has yet been identified. The Prediction of Metabolic Syndrome in Adolescence (PREMA) study was conducted (1) to construct a classification score to detect children at high risk for MetS in adolescence and (2) to test its predictive ability.

Methods: In the derivation cohort (1,270 children), data from natal and parental profiles and from initial laboratory assessment at 6-8 years of age were used to select independent predictors of MetS at 13-15 years of age, as defined by the International Diabetes Federation criteria. In the independent validation cohort (1,091 adolescents), the discriminatory capacity of the derived prediction score was tested.

Results: MetS was diagnosed in 105 adolescents in the derivation cohort $(8 \%)$. Independently, predictors of adolescent MetS were: low birth weight $<10$ th percentile (odds ratio 6.02; 95\% CI 2.53-10.12, p < $0.001)$, small birth head circumference $<10$ th percentile $(4.15 ; 2.04-7.14, \mathrm{p}<0.001)$, and at least one parent overweight or obese $(3.22 ; 1.30-5.29, \mathrm{p}<0.01)$. In the validation cohort, 86 developed MetS $(8 \%)$. These three factors predicted MetS with a sensitivity of $91 \%$ and a specificity of $98 \%$.

Conclusions: The coexistence of low birth weight, small birth head circumference, and parental history of overweight or obesity predicts infants at risk of developing MetS in adolescence.

Metabolic syndrome (MetS) is a cluster of interconnected factors that increases the risk of cardiovascular atherosclerotic diseases and T2DM. Its main components are dyslipidemia (elevated triglycerides and low high-density lipoproteins (HDL), elevation of arterial blood pressure (BP) and dysregulated glucose homeostasis, abdominal obesity and insulin resistance. The International Diabetes Federation criteria are: abdominal obesity (waist circumference $\geq 90$ th percentile) combined with at least two of the following characteristics: hypertension (systolic pressure $\geq 130 \mathrm{~mm} \mathrm{Hg}$ or diastolic pressure $\geq 85 \mathrm{~mm} \mathrm{Hg}$ ), fasting plasma glucose $\geq 100 \mathrm{mg} / \mathrm{dl}$, triglycerides $\geq 150 \mathrm{mg} / \mathrm{dl}$, and HDL cholesterol $<40 \mathrm{mg} / \mathrm{dl}$. Known risk factors for MetS include family history, poor diet, and inadequate exercise. The value of early recognition of risk factors of the MetS is clear.

After assessment of 2,361 white children and adolescents over a 10-year period, the present study showed that birth measurements and family history of obesity proved to be good predictors of future MetS. Prevalence of MetS increased with the number of risk factors, from $0 \%$ for those with no risk factors to $81 \%$ for those with all three risk factors. Combining all three predictors provided high sensitivity and specificity with a positive predictive value over $75 \%$ and a negative predictive value of $99 \%$. These findings allow the very early identification of high-risk individuals for future targeted early life interventions. 


\title{
Chemerin as a mediator between obesity and vascular inflammation in children
}

\author{
Landgraf K, Friebe D, Ullrich T, Kratzsch J, Dittrich K, Herberth G, Adams V, Kiess W, Erbs S, Korner A \\ Pediatric Research Center, Department of Women's and Child Health, University of Leipzig, Leipzig, Germany \\ Antje.Koerner@medizin.uni-leipzig.de \\ J Clin Endocrinol Metab 2012;97:E556-564
}

Background: The chemoattractant protein chemerin is expressed in adipose tissue. This study aimed to evaluate the association of chemerin with obesity and early-onset metabolic and vascular sequelae in children.

Methods: Serum chemerin concentrations were measured in 69 lean and 105 obese children and were related to their metabolic and cardiovascular parameters. In addition, a potential direct effect of chemerin on the expression of endothelial adhesion molecules and cell viability was assessed in human coronary artery endothelial cells in vitro.

Results: Chemerin concentrations were significantly higher in obese compared to lean children and were positively correlated with BMI SD score, leptin, and skinfold thickness. Moreover, significant associations were identified with the inflammatory markers: high-sensitive C-reactive protein and white blood cell count, and with markers of endothelial activation: intercellular adhesion molecule-1 (ICAM-1) and E-selectin. Multiple regression analyses confirmed chemerin as the strongest predictor of ICAM-1 and E-selectin, independent of BMI SD score. Chemerin induced ICAM-1 and E-selectin expression in endothelial cells in vitro, whereas VCAM-1 and eNOS expression and endothelial cell viability were unaffected.

Conclusion: These findings support a role for chemerin as a molecular link between fat mass and an early atherogenic risk profile in obese children.

The chemerin gene, also known as retinoic acid receptor responder 2 (RARRES2), was originally identified to be associated with psoriatic skin lesions. It is a secreted ligand of the orphan G-proteincoupled receptor chemokine-like receptor (CMKLR) [7]. The chemerin protein is a chemoattractant that promotes the recruitment of various cell types involved in innate and adaptive immunity to lymphoid organs and sites of tissue injury. In parallel, chemerin expression and secretion was found to increase dramatically with adipogenesis, while reduced chemerin expression in preadipocytes severely impairs differentiation into mature adipocytes. Beyond this autocrine function in adipocytes, chemerin/CMKLR1 signaling might have paracrine functions within adipose tissue by contributing to obesity-related inflammation.

Among adults, plasma chemerin levels have been shown to correlate positively with body mass index (BMI), fasting glucose, fasting serum insulin, plasma triglycerides, and total serum cholesterol, and to correlate negatively with high-density lipoprotein (HDL) cholesterol. Unlike the findings in adult studies, the current study shows that in children, the correlation of chemerin with parameters of glucose and insulin metabolism, as well as with HDL-C and triglycerides and blood pressure, were due to underlying obesity. This suggests that the children in this study represent an earlier stage of secondary morbidity of obesity. Separate from BMI, chemerin levels in children correspond directly to CRP, the number of white blood cells. This suggests that chemerin levels play a role in low-grade systemic inflammation. To investigate a direct link between chemerin and vascular inflammation, chemerin levels were analyzed in association with measures of endothelial activation. Endothelial inflammatory markers such as intracellular adhesion molecule-1 (ICAM-1) and E-selectin were found to be associated with chemerin levels independent from the degree of obesity. This finding suggests that chemerin plays a role in early vascular inflammation.

In recent years, it has become clear that obesity is commonly associated with chronic low-grade systemic inflammation. The chemerin protein may be one of the mediators between the accumulation of fat mass and the induction of early atherogenesis in obese children. 


\section{Sleep architecture and glucose and insulin homeostasis in obese adolescents}

Koren D, Levitt Katz LE, Brar PC, Gallagher PR, Berkowitz RI, Brooks LJ

Division of Pediatric Endocrinology and Diabetes, The Children's Hospital of Philadelphia, PA, USA

koren@email.chop.edu

Diabetes Care 2011;34:2442-2447

Background: Sleep deprivation is associated with increased risk of adult T2DM. It is uncertain whether sleep deprivation and/or altered sleep architecture affects glycemic regulation or insulin sensitivity or secretion.

Methods: In a cross-sectional observational study, 62 obese adolescents underwent oral glucose tolerance test (OGTT), anthropometric measurements, overnight polysomnography, and frequently sampled intravenous glucose tolerance test (FSIGT). $\mathrm{HbA}_{1 \mathrm{c}}$ and serial insulin and glucose levels were obtained, indices of insulin sensitivity and secretion were calculated, and sleep architecture was assessed. Correlation and regression analyses were performed to assess the association of total sleep and sleep stages with measures of insulin and glucose homeostasis, adjusted for confounding variables.

Results: There were significant U-shaped (quadratic) associations between sleep duration and both $\mathrm{HbA}_{1 \mathrm{c}}$ and serial glucose levels on OGTT and positive associations between slow-wave sleep (N3) duration and insulin secretory measures, independent of degree of obesity, pubertal stage, sex, and obstructive sleep apnea measures.

Conclusions: Both insufficient and excessive sleep were associated with short-term and long-term hyperglycemia in obese adolescents. Decreased N3 was associated with decreased insulin secretion. These effects may be interrelated, with reduced insulin secretory capacity leading to hyperglycemia. Optimizing sleep may prevent the development of T2DM in obese adolescents.

In addition to reduced physical activity and increased calorie intake, other putative causes have been suggested for the increased prevalence of obesity in recent decades [8]. These include endocrine disruptors, decreased smoking, reduction in variability of ambient temperature, pharmaceutical iatrogenesis, and a reduction in hours of sleep. The average hours of sleep have steadily decreased among US adults and children during the past several decades. Moreover, for children and adults, hours of sleep per night is inversely related to $\mathrm{BMI}$, and sleep deprivation is associated with insulin resistance, increased risk of T2DM, and higher fasting glucose among adults with preexisting diabetes.

The metabolic consequences of insufficient sleep may be the result of a lack of total sleep hours or insufficiency in a certain sleep component. We therefore need to understand the four different sleep components as defined by the American Academy of Sleep Medicine. $\mathrm{N}$ sleep (non-rapid eye movement sleep) is made up of three stages. Stage $N 1$ is an initial very brief stage when one first falls asleep and again after awakenings during the night. Each episode lasts $<10$ min. Stage N2 makes up about $50 \%$ of total sleep time in normal adult sleep. Stage N3, which is known as 'deep sleep', makes up about $20 \%$ of total sleep time in normal adult sleep. The fourth component, REM (rapid eye movement sleep), typically occupies $20-25 \%$ of total sleep. During REM, the activity of the brain is similar to activity during waking hours. The current study suggests that glucose metabolism is optimal when sleep duration is 7.5-8.5 h. The authors encourage teenagers to get more sleep and to adopt good sleep habits by avoiding caffeine and dark chocolate late in the day, and by avoiding bright lights from TV monitors or computer monitors for a few hours before they go to sleep. 


\section{Polycystic ovary-like syndrome in adolescent competitive swimmers}

Coste O, Paris F, Galtier F, Letois F, Maimoun L, Sultan C

Département d'Hormonologie, Hôpital Lapeyronie, Centre Hospitalo Universitaire Montpellier, Université Montpellier I, Montpellier, France

Fertil Steril 2011;96:1037-1042

Background: To investigate the potential effects of intensive swimming on clinical and hormonal pubertal development in adolescent girls and to determine whether hyperandrogenism contributes to menstrual disorders.

Methods: In a cross-sectional study, 18 competitive swimmers and 18 age-matched control subjects with breast stages IV or V. Clinical, biologic, and ultrasonographic investigations were done.

Results: A high number of cases of hyperandrogenism was seen in swimmers compared with control subjects ( 11 vs. 5 with $\mathrm{T}$ level $>0.5 \mathrm{ng} / \mathrm{ml})$, as well as a higher $\mathrm{LH} / \mathrm{FSH}$ ratio $(1.5$ vs. 0.9$)$ and SHBG level (58.4 vs. $39.5 \mathrm{nmol} / \mathrm{l}$ ) and more oligomenorrhea (9 vs. 4). Half of the swimmers with hyperandrogenism presented pauci- or multifollicular ovaries determined by pelvic ultrasound. Free $T$ was not significantly different between the two groups.

Conclusions: Hyperandrogenism and oligomenorrhea may be part of the spectrum of polycystic ovary syndrome in elite swimmers. These findings suggest that hyperandrogenism may have preceded the intensive training of these swimmers and may have predisposed the choice of sport for these girls. Intensive swimming may in turn have attenuated the clinical expression of their hyperandrogenism. Follow-up after the cessation of intensive activity would be helpful to evaluate the endocrine and metabolic profiles of these swimmers.

How many times has a parent asked you 'if my son plays basketball will he be taller'? Studies in gymnasts have shown that short stature in active gymnasts is partly due to a priori selection of individuals with shorter legs rather than being the result of vigorous exercise [9]. In the current study the authors aimed to understand the reasons for menstrual dysfunction among swimmers.

Young female athletes in a wide spectrum of sports activities suffer from menstrual dysfunction. Females with athletic amenorrhea have energy imbalance due to a combination of (1) increased energy expenditure, (2) low caloric intake as a result of body dissatisfaction and increased incidence of eating disorders, and (3) psychogenic stressors due to intense training and the rigors of competition. It is not surprising that the highest prevalence of menstrual dysfunction is observed in sports that emphasize low body weight, such as running and gymnastics. The hormonal profile in these cases is characterized by dysfunction of the hypothalamic-pituitary-ovarian axis with reduced $\mathrm{GnRH}$ pulsatility and low estrogen levels. The prevalence of menstrual disorders among swimmers has been reported to range from 15 to $82 \%$.

In the current study, swimmers had lower fat mass and $50 \%$ had menstrual disorders, compared to $22 \%$ of the control group. $72 \%$ of the swimmers had a T level $>0.5 \mathrm{ng} / \mathrm{ml}$, defined as hyperandrogenism. The study concluded that the cause for menstrual disorders was not due to dysfunction of the hypothalamic-pituitary-ovarian axis, but was rather secondary to hyperandrogenism. The authors suggest that the PCO-like syndrome in these female athletes is not a coincidence. A predisposition to hyperandrogenism might orient girls toward sports such as swimming, where strength is a performance criterion. Moreover, the authors even speculate that swimming may have attenuated the clinical expression of the hyperandrogenism and decreased the risk of developing MetS. 


\section{Expert panel on integrated guidelines for cardiovascular health and risk reduction in children and adolescents: summary report}

Expert panel on integrated guidelines for cardiovascular health and risk reduction in children and adolescents Pediatrics 2011;128(supp/ 5):S213-256

\section{Pediatric screening for hypercholesterolemia in Europe}

Kusters DM, de Beaufort C, Widhalm K, Guardamagna O, Bratina N, Ose L, Wiegman A

Department of Pediatrics, Academic Medical Center, Amsterdam, The Netherlands

Arch Dis Child 2012:97:272-276

Background: Different screening strategies are currently recommended to identify children with (familial) hypercholesterolemia in order to initiate early lipid management. However, these strategies are characterized to date by low adherence by the medical community and limited compliance by parents and children.

Methods: In a literature review, the authors assessed which children should undergo screening and which children are identified through current recommended strategies. Different screening tools and strategies currently used in Europe and what is known about the negative aspects of screening are discussed.

Results and Conclusions: The authors conclude that currently recommended selective screening strategies, which are mainly based on family history, lack precision and that a large percentage of children at high risk of future coronary artery disease are not being identified. They suggest that universal screening of children between 1 and 9 years of age is likely to be most effective in terms of sensitivity and specificity to identify children with familial hypercholesterolemia. However, this concept has yet to be proven in clinical practice.

The National Heart, Lung and blood Institute (NHLB) expert panel published guidelines for pediatric care providers to reduce cardiovascular disease risks in children. These guidelines were endorsed by the American Academy of Pediatrics (AAP). The recommendations address two different goals: to prevent risk-factor development (primordial prevention), and to prevent cardiovascular disease (primary prevention). These are integrated guidelines regarding nutrition, physical activity, smoking, blood pressure, obesity and the metabolic syndrome management.

Focusing on lipid management these guidelines differ from those published by the US Preventive Services Task Force (USPSTF) 4 years ago. The current guidelines suggest the following actions be carried out: (1) universal lipid screening on all children at 9-11 years of age; (2) selective screening of children aged 2-8 years who have a family history of cardiovascular disease or dyslipidemia or risk factors such as diabetes, hypertension or medical conditions; (3) no routine screening for children aged 12-16 years of age; screen only if there is new family history or at-risk medical condition; (4) universal screening once between age 17 and 21 years, and (5) the cut-off points used to consider use of drug therapy did not change.

These recommendations were supported by level B evidence, i.e. randomized control trials or diagnostic studies with minor limitations, genetic natural history studies, and overwhelmingly consistent evidence from observational studies. However, they have been much criticized because there are no long-term trials of treatment on cardiovascular outcomes and there are no data on the safety of lipid-lowering drugs in children. The proponents of universal screening suggest that children with moderate hyperlipidemia may benefit from lifestyle intervention and children with severe hyperlipidemia would benefit from decreased morbidity.

In light of disagreements in the USA, it is interesting to read the European recommendations published in the same year by Kusters et al. The authors reviewed current practices in Europe. In the Netherlands, Norway and the UK there is an ongoing cascade-screening program for children with positive index cases with familial hyperlipidemia. In Slovenia, there is general cholesterol screening in preschool children at the age of 5 years, and in Italy a selective screening program for children with a positive family history. The authors propose a strategy of universal screening when children have rou- 
tine vaccinations between 1 and 9 years of age. Yet they admit that this concept has yet to be tested. Clinicians are advised that guidelines are based on the best available information and it is up to them whether or not to follow them. Personally, I believe that children should be tested for high cholesterol levels, which may uncover not only their own FH but also the possible risk heart disease for their parents.

\section{New concerns}

\section{Risk of incident diabetes with intensive-dose compared with moderate-dose statin therapy: a meta-analysis}

Preiss D, Seshasai SR, Welsh P, Murphy SA, Ho JE, Waters DD, DeMicco DA, Barter P, Cannon CP, Sabatine MS, Braunwald E, Kastelein JJ, de Lemos JA, Blazing MA, Pedersen TR, Tikkanen MJ, Sattar N, Ray KK

BHF Glasgow Cardiovascular Research Centre, University of Glasgow, Glasgow, UK

david.preiss@glasgow.ac.uk

JAMA 2011;305:2556-2564

Background: A recent meta-analysis demonstrated that statin therapy is associated with excess risk of developing diabetes mellitus. This study aimed to investigate whether intensive-dose statin therapy increases the risk of incident diabetes compared with moderate-dose statin therapy.

Methods: Relevant trials in a literature search of MEDLINE, EMBASE, and the Cochrane Central Register of Controlled Trials (January 1, 1996, through March 31, 2011) were identified. Unpublished data were obtained from investigators. Randomized controlled endpoint trials that compared intensive-dose versus moderate-dose statin therapy and included more than 1,000 participants who were followed up for more than 1 year were included. Trial-specific odds ratios (ORs) for incident diabetes and major cardiovascular events were calculated using random-effects model meta-analysis.

Results: In 5 statin trials with 32,752 participants without diabetes at baseline, 2,749 developed diabetes (1,449 assigned intensive-dose therapy, 1,300 assigned moderate-dose therapy, representing 2.0 additional cases in the intensive-dose group per 1,000 patient-years) and 6,684 experienced cardiovascular events $(3,134$ and 3,550, respectively, representing 6.5 fewer cases in the intensive-dose group per 1,000 patient-years) over a weighted mean \pm SD follow-up of $4.9 \pm 1.9$ years. Odds ratios were 1.12 (95\% CI 1.04-1.22; $\left.\mathrm{I}^{2}=0 \%\right)$ for incident diabetes and $0.84\left(95 \%\right.$ CI $\left.0.75-0.94 ; \mathrm{I}^{2}=74 \%\right)$ for cardiovascular events for intensive- versus moderate-dose therapy. As compared with moderate-dose statin therapy, the number needed to harm per year for intensive-dose statin therapy was 498 for incident diabetes while the number needed to treat per year for intensive-dose statin therapy was 155 for cardiovascular events.

Conclusion: In a pooled analysis of data from 5 statin trials, intensive- versus moderate-dose statin therapy increased the risk of incident diabetes but reduced the risk of cardiovascular events.

Statins effectively improve survival in subjects with acute coronary syndrome or other risk factors for atherosclerotic coronary artery disease. A decrease in LDL-C levels of $40 \mathrm{mg} / \mathrm{dl}$ translates to a $13 \%$ lower risk of death. However, studies variably report that statins increase the risk for diabetes [10]. JUPITER (Justification for the Use of Statins in Primary Prevention: An Intervention Trial Evaluating Rosuvastatin) showed that statins increased the risk of newly diagnosed diabetes by $25 \%$; on the other hand, a meta-analysis of 13 randomized trials involving 91,140 patients concluded that the risk of diabetes was only $9 \%$ higher [11]. It is interesting that there was no relationship between the degree of lowering of LDL-C levels and risk of new-onset diabetes. Risk factors for diabetes include older age, higher baseline fasting glucose levels, and higher dose of statins.

The current study now shows that intensive- versus moderate-dose statin therapy increases the risk of new-onset diabetes by $12 \%$, but reduced the risk of cardiovascular events by $16 \%$. One additional patient develops diabetes for every 3 patients protected from a major cardiovascular event. How statins increase the incidence of diabetes is still unknown. These findings shed a warning for the use of statins in children, who may require statin therapy for many decades; the risk:benefit ratio of long-term statin therapy in children should be monitored. 


\section{Effect of a monoclonal antibody to PCSK9 on LDL cholesterol}

Stein EA, Mellis S, Yancopoulos GD, Stahl N, Logan D, Smith WB, Lisbon E, Gutierrez M, Webb C, Wu R, Du Y, Kranz T, Gasparino E, Swergold GD

Metabolic and Atherosclerosis Research Center, Cincinnati, OH, USA

esteinmrl@aol.com

N Engl J Med 2012;366:1108-1118

Background: Proprotein convertase subtilisin/kexin 9 (PCSK9), one of the serine proteases, binds to lowdensity lipoprotein (LDL) receptors, leading to their accelerated degradation and to increased LDL-C levels. Three phase 1 studies of a monoclonal antibody to PCSK9 designated as REGN727/SAR236553 (REGN727) are reported.

Methods: Two randomized, single ascending-dose studies of REGN727 administered either intravenously (40 subjects) or subcutaneously (32 subjects), as compared with placebo, were performed in healthy volunteers. In addition, a randomized, placebo-controlled, multiple-dose trial in adults with heterozygous familial hypercholesterolemia who were receiving atorvastatin ( 21 subjects) and those with nonfamilial hypercholesterolemia who were receiving atorvastatin (30 subjects) (baseline LDL-C, >100 mg/dl $(2.6 \mathrm{mmol} / \mathrm{l}))$ or a modified diet alone $(10$ subjects) (baseline LDL-C, $>130 \mathrm{mg} / \mathrm{dl}(3.4 \mathrm{mmol} / \mathrm{l}))$. REGN727 doses of 50,100, or $150 \mathrm{mg}$ were administered subcutaneously on days 1, 29, and 43. The primary outcome for all studies was the occurrence of adverse events. The principal secondary outcome was the effect of REGN727 on the lipid profile.

Results: There were no discontinuations of REGN727 due to adverse events. REGN727 significantly lowered LDL-C levels in all the studies. In the multiple-dose study, REGN727 doses of 50, 100, and $150 \mathrm{mg}$ reduced measured LDL-C levels in the combined atorvastatin-treated populations to $77.5 \mathrm{mg} /$ dl $(2.00 \mathrm{mmol} / \mathrm{l}), 61.3 \mathrm{mg} / \mathrm{dl}(1.59 \mathrm{mmol} / \mathrm{l})$, and $53.8 \mathrm{mg} / \mathrm{dl}(1.39 \mathrm{mmol} / \mathrm{l})$; differences in the change from baseline were $-39.2,-53.7$, and -61.0 percentage points, respectively, as compared with placebo ( $\mathrm{p}<0.001$ for all comparisons).

Conclusions: In three phase 1 trials, a monoclonal antibody to PCSK9 reduced LDL-C levels in healthy volunteers and in subjects with familial or nonfamilial hypercholesterolemia.

\section{Safety and efficacy of a monoclonal antibody to proprotein convertase subtilisin/kexin type 9 serine protease, SAR236553/REGN727, in patients with primary hypercholesterolemia receiving ongoing stable atorvastatin therapy}

McKenney JM, Koren MJ, Kereiakes DJ, Hanotin C, Ferrand AC, Stein EA

Virginia Commonwealth University and National Clinical Research, Inc., Richmond, VA, USA

J Am Coll Cardiol 2012;59:2344-2353

Background: The primary objective of this study was to evaluate the LDL-C-lowering efficacy of 5 SAR236553/REGN727 (REGN727) dosing regimens versus placebo at week 12 in patients with LDL-C $\geq 100 \mathrm{mg} / \mathrm{dl}$ on stable atorvastatin therapy. Secondary objectives included other lipid parameters and the attainment of LDL-C treatment goals of $<100 \mathrm{mg} / \mathrm{dl}(2.59 \mathrm{mmol} / \mathrm{l})$ and $<70 \mathrm{mg} / \mathrm{dl}(1.81$ $\mathrm{mmol} / \mathrm{l})$.

Methods: This double-blind, parallel-group, placebo-controlled trial randomized 183 patients with LDL-C $\geq 100 \mathrm{mg} / \mathrm{dl}(2.59 \mathrm{mmol} / \mathrm{l})$ on stable-dose atorvastatin 10,20 , or $40 \mathrm{mg}$ for $\geq 6$ weeks to: subcutaneous placebo every 2 weeks (Q2W); REGN727 at 50, 100, or $150 \mathrm{mg}(\mathrm{Q} 2 \mathrm{~W})$, or REGN727 at 200 or $300 \mathrm{mg}$ every 4 weeks (Q4W), alternating with placebo for a total treatment period of 12 weeks.

Results: REGN727 demonstrated a clear dose-response effect on percentage LDL-C lowering for both Q2W and Q4W administration: 40, 64, and 72\% with 50, 100, and $150 \mathrm{mg}$ Q2W, respectively, and 43 and $48 \%$ with 200 and $300 \mathrm{mg}$ Q4W, compared to 5\% with placebo. REGN727 also substantially reduced non-HDL cholesterol, apolipoprotein B, and lipoprotein(a). REGN727 was generally well tolerated. One patient on REGN727 experienced a serious adverse event of leukocytoclastic vasculitis. 
Conclusions: When added to atorvastatin, PCSK9 inhibition with REGN727 further reduces LDL-C by $40-72 \%$. These additional reductions are both dose- and dosing frequency-dependent.

Familial hypercholesterolemia $(\mathrm{FH})$ is an autosomal dominant disorder that causes extreme elevations in total cholesterol and LDL-C, and occurs in approximately 1 per 500 persons worldwide. It is most often associated with loss-of-function mutations in the gene encoding the LDL receptor (LDLR), and familial defective Apo-B100 (FDB) caused by mutations in ApoB-100 (APOB) that disrupt the binding of LDL to its receptor. In 2003, proprotein convertase subtilisin kexin type 9 (PCSK9) was discovered as the third class of mutations involved in autosomal dominant FH [12]. PCSK9 is synthesized primarily in the liver, and enters the circulatory system, where it binds to and accelerates the degradation of hepatic LDL receptors. This process reduces the capacity of the liver to remove LDL-C from the circulation. Patients with PCSK9 gain-of-function mutations have high LDL-C levels whereas patients with PCSK9 loss-of-function mutations have low levels of LDL-C and low incidence of coronary heart disease. Based on this knowledge, pharmacologic inhibition of PCSK9 is sought either by blocking its action or preventing its formation.

Stein et al. report phase 1 data on REGN727, a monoclonal antibody specific to PCSK9 which reduced LDL-C levels by $73 \%$ compared to a placebo in healthy volunteers and in statin-treated subjects with familial and nonfamilial forms of hypercholesterolemia. The short duration of this trial limited the ability to evaluate its safety profile. McKenney et al. confirm in a phase 2 trial that REGN727 reduced LDL cholesterol levels by $39.6 \%$ with the $50-\mathrm{mg}$ dose to $72 \%$ on $150 \mathrm{mg}$. The biweekly injection was more potent than four weekly. At week 12,100\% of the patients treated with a 150-mg injection of REGN727 had LDL-C levels $<70 \mathrm{mg} / \mathrm{dl}$. One patient developed diarrhea and a rash, which was diagnosed as leukocytoclastic vasculitis and was considered to be a significant medical event. Six other patients discontinued the drug owing to adverse events such as neutropenia, fatigue, rash, headache, and nausea.

Although concerns have been raised regarding the cost of PCSK9-directed antibodies, it is clear that this new drug is a landmark in the treatment for $\mathrm{FH}$.

\section{Important for clinical practice}

\section{Pregnancy outcomes in familial hypercholesterolemia: a registry-based study}

Toleikyte I, Retterstol K, Leren TP, Iversen PO

Department of Nutrition, Institute of Basic Medical Sciences, University of Oslo, Oslo, Norway

Circulation 2011;124:1606-1614

Background: Women with familial hypercholesterolemia (FH) are prone to early cardiovascular disease and death. It is unknown whether FH adversely affects pregnancy and birth outcomes. This study aimed to determine whether heterozygous FH women are at higher risk of premature birth $(<37$ gestational weeks), delivering infants with low birth weight $(<2,500 \mathrm{~g})$ and/or congenital malformations.

Methods: Information from the Medical Genetics Laboratory was linked to the Medical Birth Registry of Norway. 1,869 FH women ( $\geq 14$ years) from the Medical Genetics Laboratory and about 2 million (general population) from the Medical Birth Registry of Norway during the period 1967-2006 were included. The registry match allowed analysis of 2,319 births in 1,093 women with heterozygous FH. Results: The mean \pm SD prepregnancy total cholesterol concentration was $9.59 \pm 2.06 \mathrm{mmol} / \mathrm{l}(370 \pm 80$ $\mathrm{mg} / \mathrm{dl}$ ). The frequencies of prematurity, low birth weight, and congenital malformations in the $\mathrm{FH}$ population were $6.8,5.0$, and $3.3 \%$, respectively, compared to $6.2,5.2$, and $3.2 \%$ in the general population. The corresponding odds ratios were 1.11 (95 CI 0.94-1.31; $\mathrm{p}=0.23$ ), $0.96(0.79-1.15 ; \mathrm{p}=$ $0.64)$, and $1.09(0.87-1.37 ; \mathrm{p}=0.45)$.

Conclusions: Women with FH do not have higher risks of adverse pregnancy outcomes.

During pregnancy, plasma cholesterol may increase by $25-50 \%$ and plasma triglycerides by $50-300 \%$. Women with heterozygous familial hypercholesterolemia $(\mathrm{FH})$ show similar relative changes, but 
higher absolute changes, in plasma lipid levels than in healthy women. Moreover, lipid-lowering drugs are not recommended during preconception, pregnancy and lactation. Hyperlipidemia during pregnancy may induce atherosis in the uteroplacental spiral arteries that, combined with hypercoagulation, may result in thrombosis and placental infarctions, leading to placental insufficiency and thereby fetal compromise.

In this retrospective study, no maternal cardiovascular deaths were observed. Also, children of mothers with FH were no more likely than the general population to be born prematurely, have low birth weight, or have congenital malformations. The accompanying editorial [13] emphasized that high LDL-C levels during childbearing years may have long-term implications for women with $\mathrm{FH}$.

While no congenital malformations were observed in the 19 pregnancies associated with the use of lipid-lowering drugs, statins are classified as category $X$ in pregnancy, which means they have been linked to fetal abnormalities in animal and human studies, and their benefits do not outweigh their potential risks. Statin exposure during the first trimester is associated with defects of the central nervous system and unilateral limb deficiencies. The Food and Drug Administration classifies ezetimibe and niacin as category $\mathrm{C}$ in pregnancy, which means 'animal reproduction studies have shown an adverse effect on the fetus and there are no adequate and well-controlled studies in humans, but potential benefits may warrant use of the drug in pregnant women despite potential risks'.

\section{Food for thought}

\section{Improbable research: the German beer belly is misunderstood}

Abrahams M

Mini-Annals of Improbable Research 'mini-AIR' April 2012

I encourage you to check the mini-AIR website: http://www.improbable.com/airchives/miniair/. It is the simplest way to keep informed about Improbable and Ig Nobel news and events. It is advertised as 'the magazine about research that makes people laugh and then think, and it will bring a smile to your face'.

The above review cites work by scientists who questioned whether beer is the main cause of beer bellies in Germans [14]. The weight, waist and hip circumferences of 19,941 men and women were recorded related to self-reported surveys of beer consumption. Their estimates were based on the size of a typical bottle of beer in Germany. Women were placed in four categories from 'no beer' to 'moderate drinkers'. Men were placed in five categories from 'no beer' to 'heavy drinkers'. For women, 'moderate' meant consuming at least $250 \mathrm{ml}$ of beer a day. For men, 'moderate' meant 500-1,000 ml/day. The authors conclude that their study 'does not support the common belief of a site-specific effect of beer on the abdomen, the beer belly'. So now, enjoy Gose, a top-fermented beer characteristic of Leipzig. Cheers. ...!

References

1. Hegele RA, Zinman B, Hanley AJ, Harris SB, Barrett PH, Cao H: Genes, environment and Oji-Cree type 2 diabetes. Clin Biochem 2003;36:163-170.

2. Millar K, Dean HJ: Developmental origins of type 2 diabetes in aboriginal youth in Canada: it is more than diet and exercise? J Nutr Metab 2012;2012:127452.

3. Cutfield WS, Wilton P, Bennmarker H, et al: Incidence of diabetes mellitus and impaired glucose tolerance in children and adolescents receiving growth-hormone treatment. Lancet 2000;355:610-613.

4. Flint A, Arslanian S: Treatment of type 2 diabetes in youth. Diabetes Care 2011;34(suppl 2):S177-183.

5. Sakhaee K, Maalouf NM, Sinnott B: Kidney stones 2012: pathogenesis, diagnosis, and management. J Clin Endocrinol Metab 2012.

6. Eknoyan G: Michelangelo: art, anatomy, and the kidney. Kidney Int 2000;57:1190-1201.

7. Ernst MC, Sinal CJ: Chemerin: at the crossroads of inflammation and obesity. Trends Endocrinol Metab 2010;21:660667.

8. Keith SW, Redden DT, Katzmarzyk PT, et al: Putative contributors to the secular increase in obesity: exploring the roads less traveled. Int J Obes (Lond) 2006;30:1585-1594. 
9. Bass S, Bradney M, Pearce G, et al: Short stature and delayed puberty in gymnasts: influence of selection bias on leg length and the duration of training on trunk length. J Pediatr 2000;136:149-155.

10. Goldfine AB: Statins: is it really time to reassess benefits and risks? N Engl J Med 2012;366:1752-1755.

11. Sattar N, Preiss D, Murray HM, et al: Statins and risk of incident diabetes: a collaborative meta-analysis of randomised statin trials. Lancet 2010;375:735-742.

12. Abifadel M, Varret M, Rabes JP, et al: Mutations in PCSK9 cause autosomal dominant hypercholesterolemia. Nat Genet 2003;34:154-156

13. Rutherford JD: Maternal heterozygous familial hypercholesterolemia and its consequences for mother and child. Circulation 2011;124:1599-1601.

14. Schutze M, Schulz M, Steffen A, et al: Beer consumption and the 'beer belly': scientific basis or common belief? Eur J Clin Nutr 2009;63:1143-1149. 